\title{
Neurosurgical publications in China: an analysis of the web of science database
}

\author{
Weiming Liu*, Deling Li, Ming Ni, Wang Jia, Weiqing Wan, Jie Tang and Guijun Jia
}

\begin{abstract}
Background: Neurosurgery in China has made great progress. The aim of this study was to analyze neurosurgical publications by Chinese authors using the Web of Science database as a way to illustrate the current state of neurosurgery in China.

Methods: We searched the Web of Science core database for articles containing "neurosurg*" with or without an author address in "China" to obtain the set of neurosurgical publications in China and worldwide. We then extracted data from the search results to obtain information such as document type, countries/territories, organizations, publication year, title and research area. Then, we analyzed the search results of articles (document type) to generate a citation report. We identified publications by Chinese neurosurgeons that were cited more than 100 times.

Results: A total of 165,365 neurosurgical publications were identified. Among them, 10,770 were published by Chinese neurosurgeons. Chinese neurosurgical publications have increased year by year, accounting for $2 \%$ of neurosurgical publications before 2010 and rising to $13 \%$ from 2010 to the present. The most frequent journals for Chinese neurosurgeons differ from the most frequent journals worldwide. We identified 34 Chinese organizations that published more than 100 publications. We also identified 19 studies written by Chinese neurosurgeons that were cited more than 100 times. Basic research represents a large proportion of Chinese publications in this area, while clinical research remains a weak area. Cooperative studies were overrepresented among Chinese studies.

Conclusion: While China's publication output in the area of neurosurgery has made great progress, significant room for improvement remains. The next step is to strengthen Chinese neurosurgical clinical studies and improve the publishing environment for Chinese neurosurgeons.
\end{abstract}

Keywords: Neurosurgery, Publications, China, Web of science

\section{Background}

For historical reasons, the field of neurosurgery has developed in China along its own unique path [1,2]. Today's China is more open than ever, including in academic research. Chinese neurosurgeons need to learn from their foreign counterparts, while doctors outside China also need to know what Chinese doctors have done. Publications represent the best bridge between these groups. In recent years, China doctors have begun to realize the importance of publication, and have begun to capture their discoveries and experience in publications to communicate their findings to others. As the main international academic language is English, Chinese neurosurgeons have to publish their work in English to reach worldwide recognition. As a result, language limitations obstruct academic exchanges between Chinese doctors and those from other countries.

Chinese neurosurgeons have published a significant body of work. Thus far, Chinese neurosurgery has made significant achievements in both clinical practice and scientific research, obtaining worldwide recognition and respect. As China has the largest population in the world, it should represent the largest disease resource database, and the voices of Chinese physicians should be well represented in the academic discussion. The first task is to understand the work that has already been done by Chinese neurosurgeons and China's current position in the field.

\footnotetext{
* Correspondence: dr_wmliu@hotmail.com

Department of Neurosurgery, Beijing Tiantan Hospital, Capital Medical

University, Tiantan Xili 6, Dongcheng District, Beijing 100050, China
} 
The most commonly used medical biological databases-PubMed, Web of Science, and Google Scholar-each have their own strengths and weaknesses [3]. Among them, Web of Science (WoS) is a strong research database that was officially inaugurated in 2004 by the Thomson Scientific and Health Care Corporation. The WoS database not only contains the affiliations of all authors but also provides the numbers of citations of published articles. WoS provides access to Thomson Reuter's multidisciplinary databases of bibliographic information. WoS is a powerful web interface providing access to the citation database [4]. WoS offers a significant advantage for literature reviews in some particular fields $[5,6]$, especially for efforts to understand overall trends $[7,8]$. Previous studies have evaluated Chinese neurosurgical publications, but they only focused on local Chinese journals [9] or carried out only superficial research on Chinese neurosurgical publications [10]. The aim of this study was to comprehensively analyze neurosurgical publications in China using the WoS database.

\section{Methods}

The Web of Science (WoS) database was accessed on May 4, 2015. Publications in the Web of science core collection database were searched for publications of all types with authors' addresses containing "neurosurg*" or "surgical neurology". The number of publications and other information was obtained. In the first stage, an international comparison of neurosurgeons' research outputs by country was carried out. Then, the search was divided into two periods, before 2010 (1928-2009) and after 2010 (2010 to present) to extract more detailed information.

Chinese neurosurgical publications were identified using the following search strategy: ((address: neurosurg*) OR (address: surgical neurology)) AND (address: China). Data were extracted from the search results to obtain information such as document type, countries/territories, funding agencies, organizations, publication year, title and research area. Then, the search results were analyzed using the "analyze results" tool on the WoS webpage.

We only included articles in the citation report, excluding reviews, meeting abstracts, letters, editorial material and other document types. We searched for papers with Chinese corresponding authors cited more than 100 times to identify Chinese neurosurgeons playing important roles in research. We also spoke with senior experts in the neurosurgical field and manually retrieved some original documents to ensure a complete survey.

Publications from the departments/institutes of neurosurgery in China and data sources from the Web of science core collection database were searched. The types of publications included articles, letters, reviews, proceedings papers, editorial materials, and more. Analyses

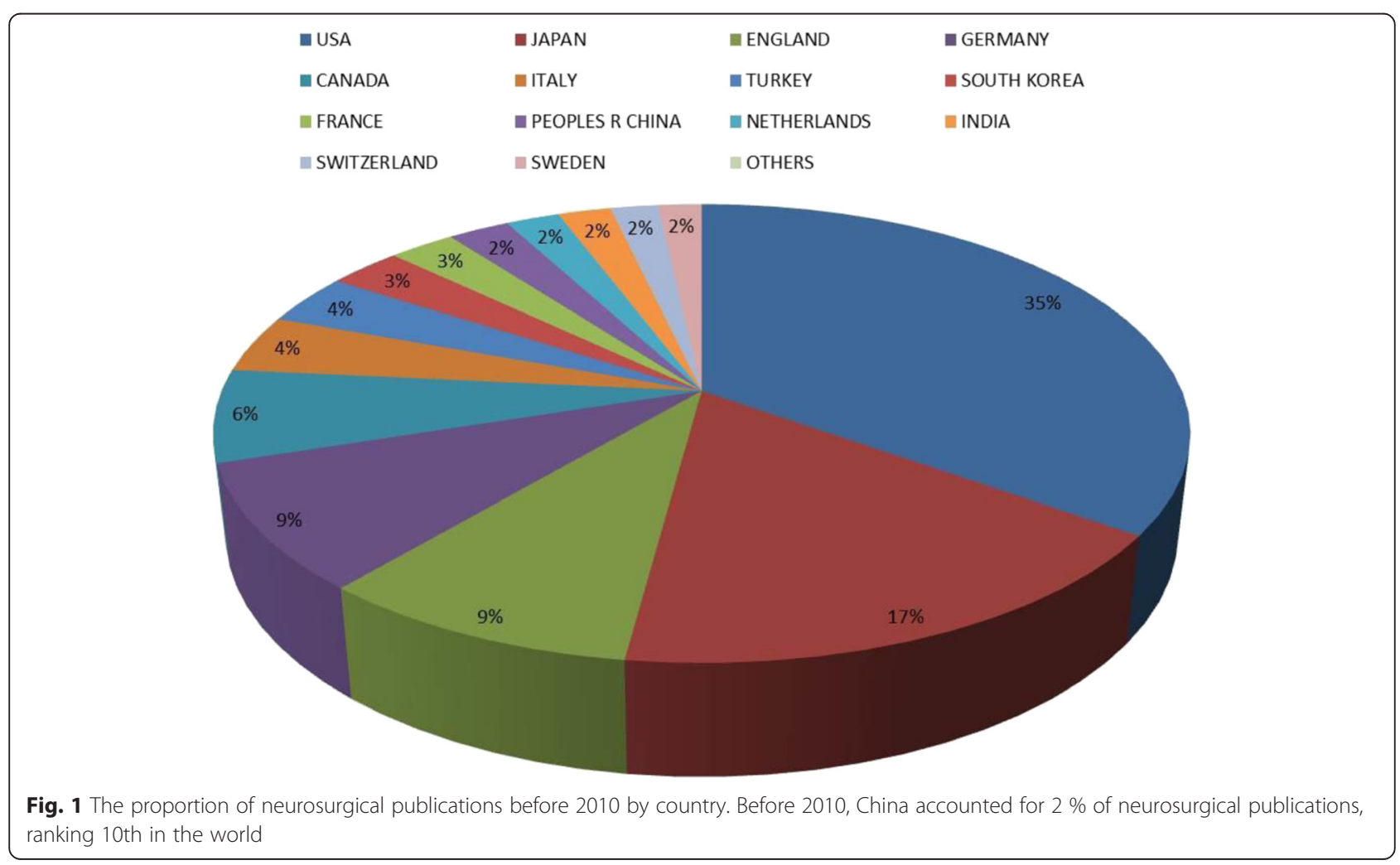




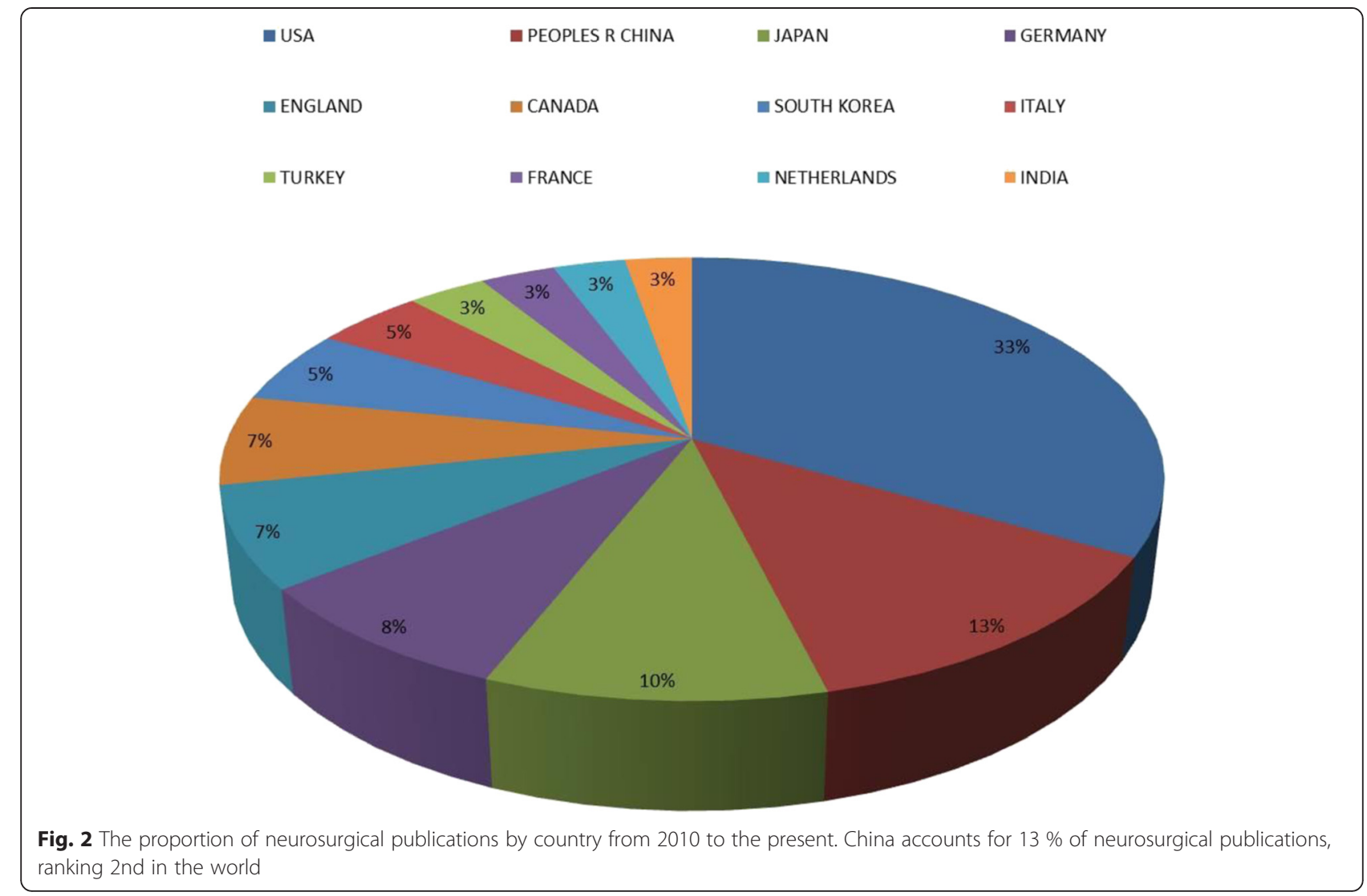

included yearly research output, research output over the whole time period, WoS subject category, authors, organizations/institutes that submitted published articles, and the names of journals publishing articles.

Data from WoS were exported to a Microsoft Office ${ }^{\bullet}$ Excel spreadsheet and then transferred to a Microsoft Word document. We considered the top ranked publications in each item. ANOVA, Student's t-, and c2 tests were used to assess the statistical significance of differences in the mean citation numbers among different study periods (SPSS version 17.0; SPSS Inc., Chicago,
IL, USA). A $p$ value $<0.05$ was considered statistically significant (2-tailed test).

\section{Result}

Proportional growth in Chinese publications

Worldwide, neurosurgical departments have been involved in a total of 165,365 publications, 61,411 of which were published since the beginning of 2010. Before 2010, Chinese neurosurgeons published only $2 \%$ of total publications, ranking tenth in the world (Fig. 1). Chinese neurosurgical authors contributed $13 \%$ of publications in the

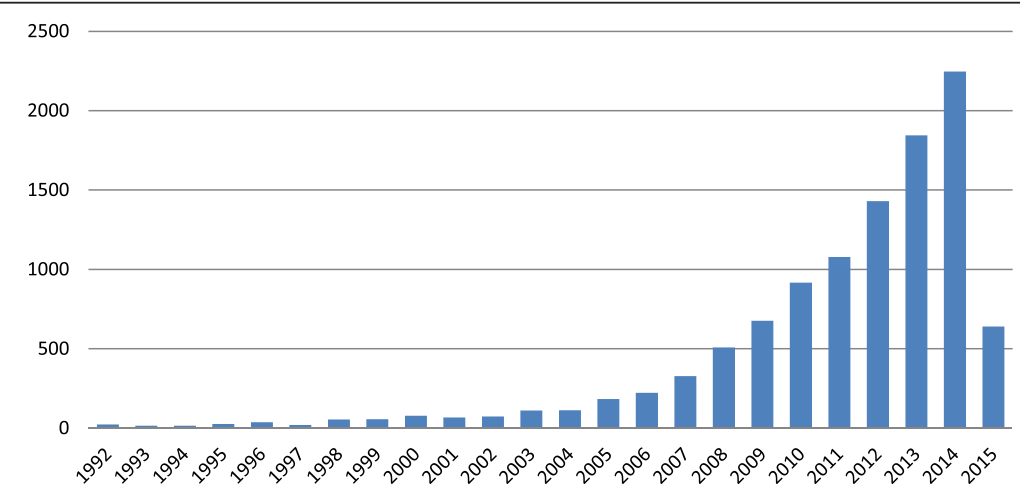

Fig. 3 The number of Chinese neurosurgical publications per year has increased rapidly 


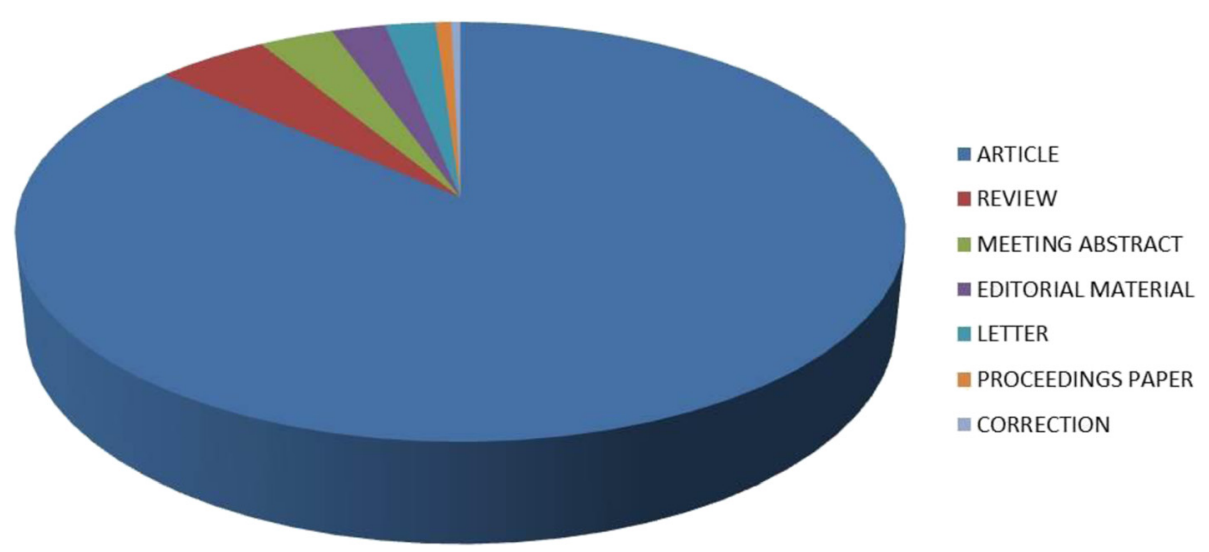

Fig. 4 Document types of Chinese publications

period after 2010, rising to the second most productive country in the world (Fig. 2).

Chinese neurosurgeons have published a total of 10,770 publications. The number of publications has increased every year, with especially rapid growth observed in recent years (Fig. 3).

The greatest proportion of these publications are articles, accounting for more than $87 \%$, far more than other document types (Fig. 4).

\section{Details of Chinese neurosurgical publications}

The 10 journals most frequently publishing articles by Chinese neurosurgeons differ from those publishing articles by neurosurgeons from throughout the world (Table 1). Chinese neurosurgeons have published few articles in the most famous magazines in the field of neurosurgery, such as "Journal of Neurosurgery" and "Neurosurgery". Instead, they have frequently published papers in general medical journals such as "Chinese Medical Journal" and "PLOS One". Journals emphasizing basic research have also published many articles by Chinese neurosurgeons.
There are 34 Chinese organizations that have published more than 100 publications in this research area (Table 2). The most prolific is "Capital Medical University", which has published 1287 publications, accounting for $12 \%$ of all Chinese neurosurgical publications. Most of these organizations are universities located in central cities of Eastern China.

We identified 19 studies written by Chinese authors that have been cited more than 100 times (18 were identified from a database search, while 1 came from consulting a senior expert). The details of these studies are shown in Table 3. Most of these studies are basic research. However, some report research on clinical practice such as brain trauma, brain stem cavernous malformations, and epidemiological investigations in China. Because of China's huge population and number of patients, the experiences and discoveries of Chinese neurosurgeons are an important part of the global knowledge base.

\section{Research area trends}

Comparing publications before and after 2010, the distribution of worldwide publications among different research

Table 1 The top 10 journals publishing Chinese's and world neurosurgeon's publications

\begin{tabular}{|c|c|c|c|c|c|}
\hline \multicolumn{3}{|l|}{ Chinese neurosurgeon publications } & \multicolumn{3}{|l|}{ World neurosurgeon publications } \\
\hline Source Titles & Records & $\%$ of all & Source Titles & Records & $\%$ of all \\
\hline Chinese medical journal & 436 & 4.048 & Journal of neurosurgery & 7211 & 4.361 \\
\hline Plos ONE & 309 & 2.869 & Neurosurgery & 6626 & 4.007 \\
\hline Journal of clinical neuroscience & 295 & 2.739 & Acta neurochirurgica & 4785 & 2.894 \\
\hline Neural regeneration research & 239 & 2.219 & Surgical neurology & 3351 & 2.026 \\
\hline Brain research & 199 & 1.848 & Childs nervous system & 3158 & 1.91 \\
\hline Journal of neuro oncology & 184 & 1.708 & Neurologia medico chirurgica & 3082 & 1.864 \\
\hline Acta neurochirurgica & 157 & 1.458 & Journal of clinical neuroscience & 2876 & 1.739 \\
\hline Neuroscience letters & 153 & 1.421 & Neuro oncology & 2542 & 1.537 \\
\hline Clinical neurology and neurosurgery & 145 & 1.346 & Epilepsia & 2465 & 1.491 \\
\hline Neurological research & 143 & 1.328 & Journal of neuro oncology & 2385 & 1.442 \\
\hline
\end{tabular}


Table 2 Organizations who published more 100 publications in China

\begin{tabular}{|c|c|c|c|}
\hline & Organizations & Records & $\%$ of all \\
\hline 1 & Capital Medical University & 1287 & 11.95 \\
\hline 2 & Fudan University & 776 & 7.205 \\
\hline 3 & Beijing Neurosurgical Institute & 667 & 6.193 \\
\hline 4 & Shanghai Jiao tong University & 666 & 6.184 \\
\hline 5 & Fourth Military Medical University & 594 & 5.515 \\
\hline 6 & China Medical University & 540 & 5.014 \\
\hline 7 & Sichuan University & 431 & 4.002 \\
\hline 8 & Zhejiang University & 413 & 3.835 \\
\hline 9 & Shandong University & 386 & 3.584 \\
\hline 10 & Second Military Medical University & 375 & 3.482 \\
\hline 11 & Chinese University Of Hong Kong & 343 & 3.185 \\
\hline 12 & Harbin Medical University & 334 & 3.101 \\
\hline 13 & Third Military Medical University & 331 & 3.073 \\
\hline 14 & China Medical University Taiwan & 330 & 3.064 \\
\hline 15 & Tianjin Medical University & 312 & 2.897 \\
\hline 16 & Nanjing Medical University & 308 & 2.86 \\
\hline 17 & Southern Medical University China & 307 & 2.851 \\
\hline 18 & Sun Yat Sen University & 284 & 2.637 \\
\hline 19 & Suzhou University & 278 & 2.581 \\
\hline 20 & Jilin University & 272 & 2.526 \\
\hline 21 & Nanjing University & 257 & 2.386 \\
\hline 22 & Prince Of Wales Hospital & 248 & 2.303 \\
\hline 23 & Chinese Academy of Sciences & 242 & 2.247 \\
\hline 24 & China Medical University Hospital Taiwan & 242 & 2.247 \\
\hline 25 & $\begin{array}{l}\text { Chinese Academy Of Medical Sciences Peking } \\
\text { Union Medical College }\end{array}$ & 239 & 2.219 \\
\hline 26 & Huazhong University of Science Technology & 216 & 2.006 \\
\hline 27 & Central South University & 212 & 1.968 \\
\hline 28 & Peking University & 206 & 1.913 \\
\hline 29 & Xi An Jiaotong University & 158 & 1.467 \\
\hline 30 & Tongji University & 125 & 1.161 \\
\hline 31 & University of Hong Kong & 122 & 1.133 \\
\hline 32 & Chongqing Medical University & 119 & 1.105 \\
\hline 33 & Nantong University & 117 & 1.086 \\
\hline 34 & Fujian Medical University & 113 & 1.049 \\
\hline
\end{tabular}

areas has changed somewhat. The following research areas have increased in proportion between the two periods: oncology, research experimental medicine, orthopedics, general internal medicine, cardiovascular system, cardiology, cell biology, pharmacology, and pharmacy.

At the same time, the proportions of the following research areas have decreased: neurosciences, neurology, surgery, radiology, nuclear medicine, medical imaging, pediatrics, pathology, psychiatry and endocrinology metabolism. However, these changes are not statistically significant (Fig. 5).

As for Chinese publications, basic research areas such as cell biology, pharmacology, pharmacy, biochemistry, and molecular biology represent a higher proportion of publications compared with other countries. Clinical research areas such as neurosciences, neurology, and surgery are underrepresented among Chinese publications compared with the rest of the world (Fig. 5).

\section{International collaborative research}

Among 10,770 Chinese publications, 9385 are articles (not including reviews, meeting abstracts, letters and editorial materials). The studies by Chinese neurosurgeons represent collaborations with researchers from more than 20 countries, mostly developed countries such as the United States, Japan, Canada, Britain, Germany, and Australia (Fig. 6).

The citation report from these 9385 articles is displayed in Table 4. Among the 9385 articles, 2393 were the products of international collaborations. We find that these collaborative publications have a greater impact factor than the overall average impact factor. The average Citations per Item across all Chinese publications in this sample is 8.23, while the collaborative publications' Average Citations per Item is 15.46. The h-index of all publications (85) reflects the fact that nearly 7000 of these articles do not include statistics. The h-index for the collaborative publications (76) was not significantly reduced. Overall, these results suggest that the collaborative publications have a higher average quality.

\section{Discussion}

For historical reasons, Chinese neurosurgeons have only recently begun to publish their work in English language publications. Compared with foreign neurosurgeons, Chinese neurosurgeons have not historically published prolifically. With China's development, the health care system in China has entered a new era. Chinese physicians need to update their knowledge to remain consistent with global trends, and they also want to share their findings with the world. Publications represent the ideal medium for these communications, and Chinese doctors have recognized this point.

The research output of Chinese neurosurgeons has increased every year, with a growth rate obviously higher than the worldwide average. The proportion of neurosurgical publications with Chinese authors has increased to $13 \%$, second only after the United States (35\%). Both the quality and quantity of Chinese research has improved, facilitating exchange with neurosurgeons all over the world. 
Table 3 Chinese neurosurgical publications cited more than 100

Title
1 Glioma-Derived Mutations in IDH1 Dominantly Inhibit IDH1 Catalytic Activity and Induce HIF-1 alpha. [19]

2 Stroke in China: epidemiology, prevention, and management strategies [20]

3 hsa-mir-181a and hsa-mir-181b function as tumor suppressors in human glioma cells [21]

4 Conversion of human umbilical cord mesenchymal stem cells in Wharton's jelly to dopaminergic neurons in vitro: Potential therapeutic application for parkinsonism [22]

5 Improved outcomes from the administration of progesterone for patients with acute severe traumatic brain injury: a randomized controlled trial [23]

6 Astrocyte elevated gene- 1 is a novel prognostic marker for breast cancer progression and overall patient survival [24]

7 Monodisperse water-soluble magnetite nanoparticles prepared by polyol process for highperformance magnetic resonance imaging [25]

8 Early indicators of prognosis in 846 cases of severe traumatic brain injury [26]

9 Downregulation of miR-21 inhibits EGFR pathway and suppresses the growth of human glioblastoma cells independent of PTEN status [27]

10 MicroRNA-128 inhibits glioma cells proliferation by targeting transcription factor E2F3a [28]

11 Detection of multiple gene amplifications in glioblastoma multiforme using array-based comparative genomic hybridization [29]

12 Effect of long-term mild hypothermia therapy in patients with severe traumatic brain injury: 1-year follow-up review of 87 cases [30]

13 Incidence and trends of stroke and its subtypes in China - Results from three large cities [31]

14 Influence of patients' age on functional recovery after transplantation of olfactory ensheathing cells into injured spinal cord injury [32]

15 Surgical management of brain-stem cavernous malformations: Report of 137 cases [33]

16 Clinical evaluation and follow-up outcome of diffusion Wu, Jin-Song; Zhou, Liang-Fu; Tang, Wei-Jun; tensor imaging-based functional neuronavigation: a prospective, controlled study in patients with gliomas involving pyramidal tracts. [34]

17 Poly-L-lactic acid/hydroxyapatite hybrid membrane for bone tissue regeneration [35]

18 Overexpression of astrocyte elevated gene-1 (AEG-1) is associated with esophageal squamous cell carcinoma (ESCC) progression and pathogenesis [36]

19 An in vivo evaluation of a biodegradable genipincross-linked gelatin peripheral nerve guide conduit material [37] Liu, BS

\begin{tabular}{lll} 
Authors & Source & Cited \\
\hline Zhao, Shimin; Lin, Yan; Xu, Wei; Jiang, Wenqing; & Science2009, 324:261-5 & 422 \\
Zha, Zhengyu; Wang, Pu; Yu, Wei; Li, Zhiqiang; & & \\
Gong, Lingling; Peng, Yingjie; Ding, Jianping; & &
\end{tabular}

Lei, Qunying; Guan, Kun-Liang; Xiong, Yue

Liu, Ming; Wu, Bo; Wang, Wen-Zhi; Lee, Li-Ming; Zhang, Shi-Hong; Kong, Ling-Zhi

Shi, Lei; Cheng, Zihao; Zhang, Junxia; Li, Rui; Zhao, Peng; Fu, Zhen; You, Yonyping

Fu, YS; Cheng, YC; Lin, MYA; Cheng, H; Chu, PM; Chou, SC; Shih, YH; Ko, MH; Sung, MS

The Lancet Neurology 2007, 6:456-64.

Brain research 2008, 1236:185-93.

Stem cells (Dayton, Ohio) 2006, 24:115-24.

Xiao, Guomin; Wei, Jing; Yan, Weiqi; Wang, Weimin; Critical care (London, England) 167 Lu, Zhenhui 2008, 12:R61

Li, Jun; Zhang, Nu; Song, Li-Bing; Liao, Wen-Ting; Jiang, Li-Li; Gong, Li-Yun; Wu, Jueheng; Yuan, Jie; Zhang, Hui-Zhong; Zeng, Mu-Sheng; Li, Mengfeng

Wan, Jiaqi; Cai, Wei; Meng, Xiangxi; Liu, Enzhong

Clinical cancer research : 2008, 146 14:3319-26.

Chemical communications 144 (Cambridge, England) 2007:5004-6.

Jiang, JY; Gao, GY; Li, WP; Yu, MK; Zhu, C

Journal of neurotrauma 2002, 19:869-74.

Zhou, Xuan; Ren, Yu; Moore, Lynette; Mei, Mei; You, Yongping; Xu, Peng; Wang, Baoli; Wang, Guangxiu; Jia, Zhifan; Pu, Peiyu; Zhang, Wei; Kang, Chunsheng

Zhang, Yu; Chao, Tengfei; Li, Ran; Liu, Wei; Chen, Yang; Yan, Xingqi; Gong, Yanhua; Yin, Bin; Liu, Wei; Qiang, Boqing; Zhao, Jizhong; Yuan, Jiangang; Peng, Xiaozhong

Hui, ABY; Lo, KW; Yin, XL; Poon, WS; Ng, HK

Laboratory investigation; 2010, 126 90:144-55.

Journal of molecular medicine $\quad 124$ (Berlin, Germany) 2009, 87:43-51.

Laboratory investigation; 2001, 124 81:717-23.

Jiang, JY; Yu, MK; Zhu, C

Journal of neurosurgery 2000, 93:546-9.

Jiang, B; Wang, WZ; Chen, HL; Hong, Z; Yang, QD; Stroke; 2006, 37:63-8. $\mathrm{Wu}, \mathrm{SP} ; \mathrm{Du}, \mathrm{XL} ; \mathrm{Bao}, \mathrm{QJ}$

Huang, HY; Chen, L; Wang, HM; Xiu, B; Li, BC; Wang, R; Zhang, J; Zhang, F; Gu, Z; Li, Y; Song, YL; Hao, W; Pang, SY; Sun, JZ

Wang, CC; Liu, A; Zhang, JT; Sun, B; Zhao, YL

Chinese medical journal 2003, 116:1488-91.

Surgical neurology 2003, 59:444-54; discussion 454

Neurosurgery 2007, 61: 935-48; discussion 948-9

Mao, Ying; Hu, Jin; Song, Yan-Yan; Hong,

Xun-Ning; Du, Gu-Hong

Sui, Gang; Yang, Xiaoping; Mei, Fang; Hu, Xiaoyang; Journal of biomedical Chen, Guogiang; Deng, Xuliang; Ryu, Seungkon

materials research Part A 2007 82:445-54.

Yu, Chunping; Chen, Kun; Zheng, Haiqing; Guo, Xianzhi; Jia, Weihua; Li, ManZhi; Zeng, Musheng; Li, Jun; Song, Libing

Carcinogenesis 2009, 30:894-901

Chen, YS; Chang, JY; Cheng, CY; Tsai, FJ; Yao, CH; Biomaterials 2005, 26:3911-8. 


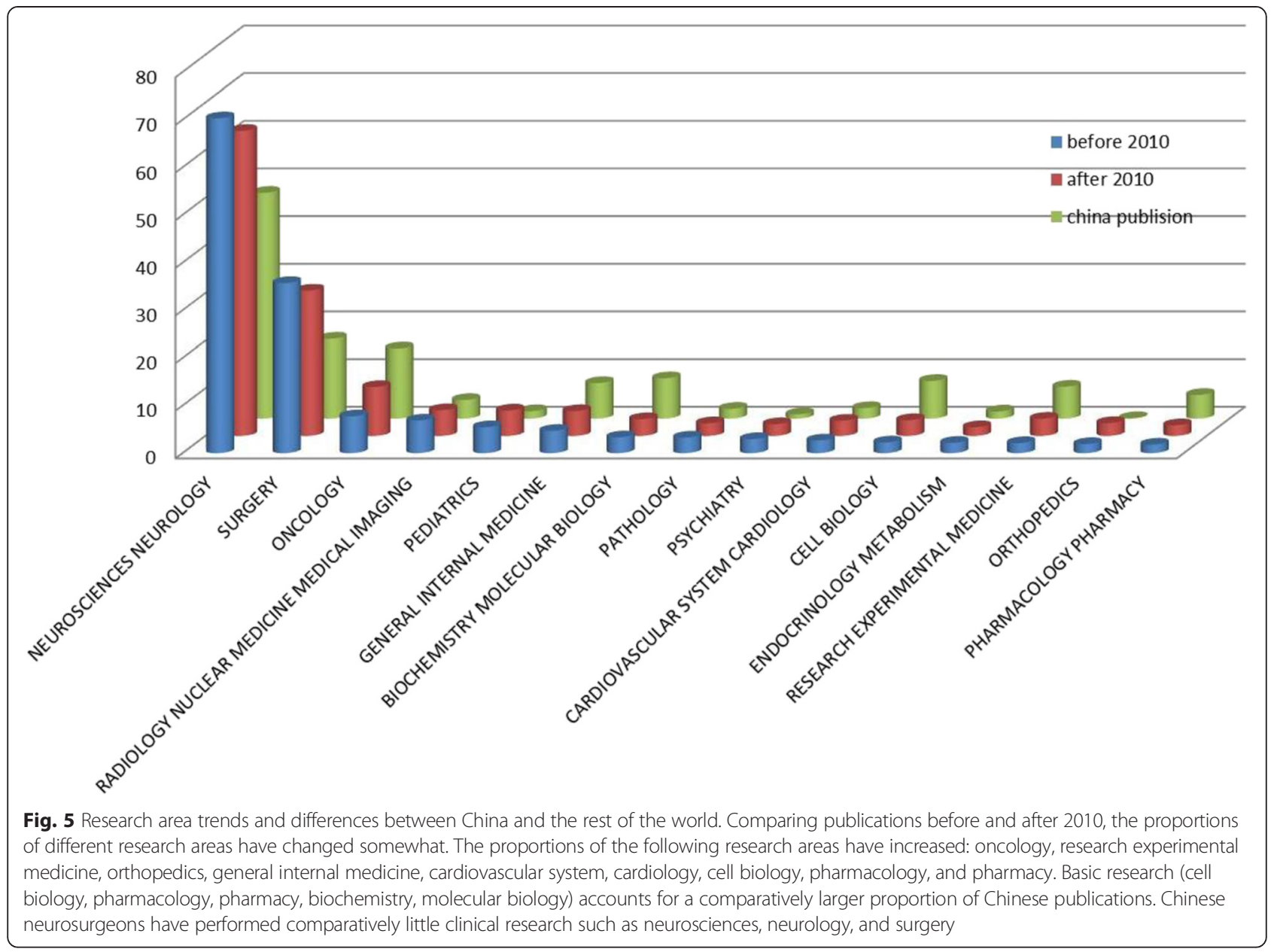

This study highlights the great gap in neurosurgery between China and developed countries. While clinical practice research is an important area of research (neurosciences, neurology, and surgery) for neurosurgeons throughout the world, this research area represents a weak point for Chinese neurosurgeons. In real clinical work, Chinese neurosurgeons also follow guidelines from developed countries. Each country's clinical practice has its own features, and issues with the implementation of clinical practice guidelines must be taken into account [11, 12]. Chinese neurosurgeons should develop guidelines in line with their evidence combined with their social and cultural environment [13]. This calls for increased attention to clinical research in the future.

China presents several advantages for clinical research. China has the largest population base, the largest pool of disease cases, and the largest group of neurosurgeons. Chinese clinical research results should be credible and widespread because of the large number of Chinese neurosurgeons. Meanwhile, we should maintain our strength in basic research, as these research results can be translated into clinical practice.
Chinese authors have infrequently published in the classic neurosurgical journals such as "Journal of Neurosurgery" and "Neurosurgery". To solve this problem, Chinese neurosurgeons need to target these journals with the aim of allowing more foreign counterparts to understand what Chinese doctors have done. Although there are a few neurosurgical journals in China, they are all published in Chinese and can only be read by Chinese readers. A direct way to expand the influence of Chinese neurosurgery is to build China's own neurosurgical publication platform. "Chinese Neurosurgical Journal" is the first Neurosurgical journal published in English in China. There is no doubt that "Chinese Neurosurgical Journal" will play an important role in introducing Chinese studies to the world.

Capital Medical University and Fudan University are the leading Chinese organizations in this field, mainly because these two universities have very well-known neurosurgical centers, such as the neurosurgical departments in Tiantan Hospital [14] and Huashan Hospital [15]. Beijing Neurosurgical Institute (number 3 in the list) is also set in Beijing Tiantan Hospital and 


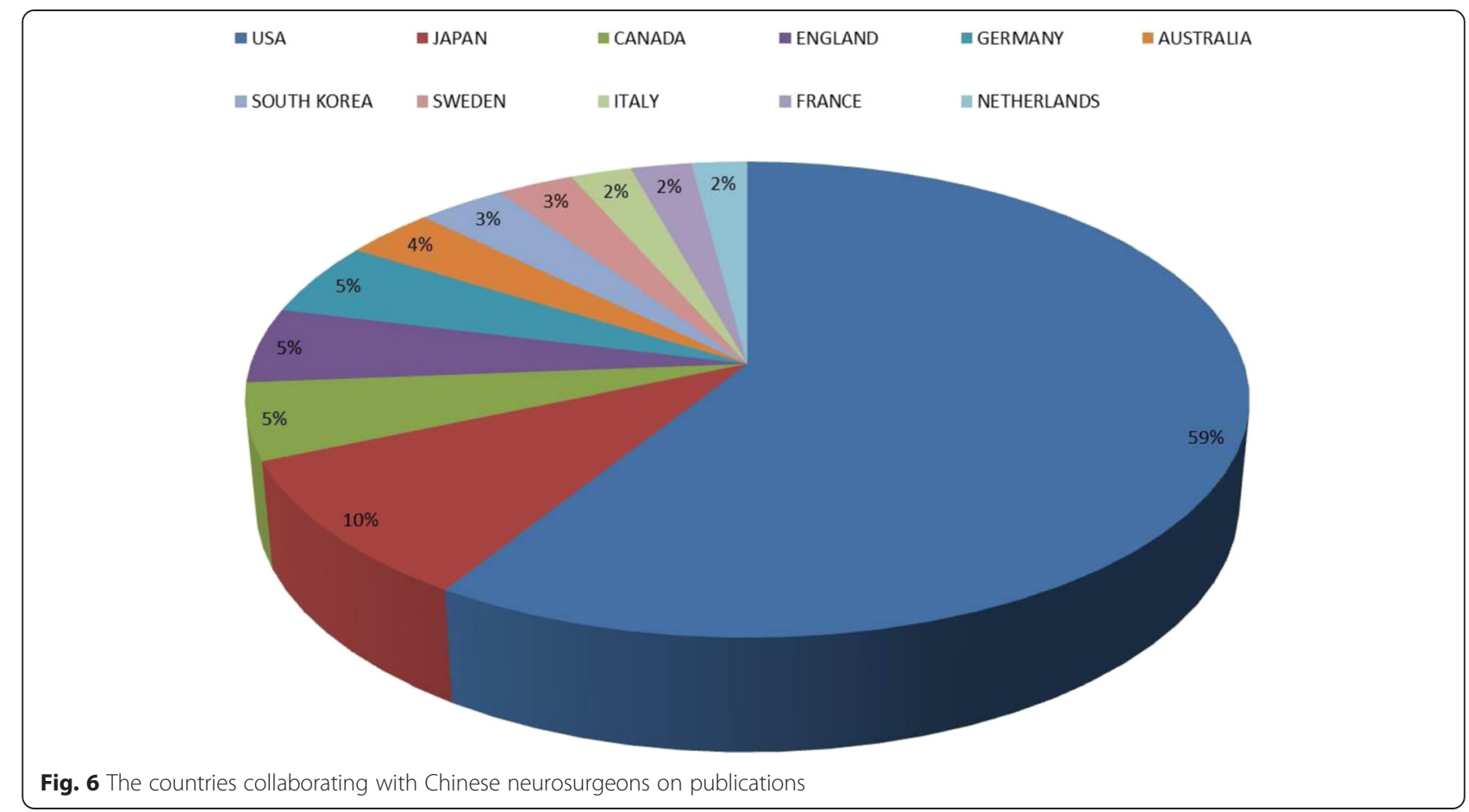

has many links with Beijing Tiantan Hospital. The list of organizations shows that most of the organizations are in central cities of eastern China, reflecting China's vast territory and unbalanced development.

China presents several advantages for clinical research. China has he largest population base, the largest pool of disease cases, and the largest group of neurosurgeons. Chinese clinical research results should be credible and widespread because of the large number of Chinese neurosurgeons.

Neurosurgeons play an unparalleled role in the development of brain research. Neurosurgery is a rapidly developing field of research, and now that Chinese neurosurgeons have begun to play a major role in this field, they should seize the opportunity and continue to publish important basic and clinical research in top journals in the field.

Table 4 Citation report of all China neurosurgeon's publications and cooperation publications

\begin{tabular}{lll}
\hline & $\begin{array}{l}\text { All Chinese } \\
\text { publications }\end{array}$ & $\begin{array}{l}\text { Cooperation } \\
\text { publications }\end{array}$ \\
\hline Results found: & 9385 & 2393 \\
Sum of the Times Cited & 77275 & 37000 \\
Citing Articles & 59272 & 35745 \\
$\begin{array}{l}\text { Citing Articles without } \\
\text { self-citations }\end{array}$ & 55306 & 31949 \\
Average Citations per Item & 8.23 & \\
h-index & 85 & 15.46 \\
\hline
\end{tabular}

\section{Limitations of this study}

While the Web of Science is an excellent database, it has several limitations. Web of Science is an Englishlanguage database, containing very little literature in other languages. This may lead to a selection bias.

Another limitation comes from scientometrics and bibliometrics. The statement that "no one metric can fully capture the complex contributions scholars make to their disciplines"[16] highlights the deficiencies of the journal impact factor (JIF) [17]. There is a growing view that for greater accuracy, the JIF must be supplemented with an article-based assessment and peer-review [18]. Therefore, this study valued article-based citations rather than the journal impact factor.

\section{Conclusion}

While Chinese neurosurgical publication has made great progress, there is still significant room for improvement. The next step is to strengthen Chinese neurosurgical clinical studies and to improve the publishing environment of Chinese neurosurgeons in an effort to introduce the results to the rest of the world.

\section{Competing interests}

All authors declare that they have no competing interests (both financial and non-financial competing interests).

\section{Authors' contributions}

Weiming Liu designed and supervised the entire study. Deling Li and Weiqing Wan searched the database. Wang Jia and Jie Tang extracted data and reported results. Ming Ni and Guijun Jia reviewed the report and 
provided recommendations on the discussion and conclusion. All authors read and approved the final manuscript.

\section{Acknowledgement}

No additional acknowledgements.

Dr. Weiming Liu received funding for this study from Capital Medical

University Basics-Clinical Research Cooperation Fund (15JL49).

Received: 19 May 2015 Accepted: 12 October 2015

Published online: 18 December 2015

\section{References}

1. Zhao J-Z. A glance at Chinese neurosurgery. Chin Med J. 2008;121:1059-60.

2. Zhao Y. A brief history of neurosurgery in the mainland of China. Zhonghua wai ke za zhi. 2015;53:33-41. Chinese journal of surgery.

3. Falagas ME, Pitsouni El, Malietzis GA, Pappas G. Comparison of PubMed, Scopus, Web of science, and Google scholar: strengths and weaknesses. FASEB J : Offi Pub Fed Am Soc Exp Bio. 2008;22:338-42.

4. Reuters T. Web of science. New York: Thomson Reuters; 2010.

5. Sweileh WM, Zyoud SH, Al-Jabi SW, Sawalha AF. Assessing urology and nephrology research activity in Arab countries using ISI web of science bibliometric database. BMC Res Notes. 2014;7:258.

6. Huh S. Citation analysis of the Korean journal of urology from Web of science, Scopus, Korean medical citation index, KoreaMed synapse, and Google scholar. Korean J Urology. 2013;54:220-8.

7. Leydesdorff L, Carley S, Rafols I. Global maps of science based on the new Web-of-Science categories. Scientometrics. 2013;94:589-93.

8. Pulgar R, Jiménez-Fernández I, Jiménez-Contreras E, Torres-Salinas D, Lucena-Martín C. Trends in World Dental Research: an overview of the last three decades using the Web of Science. Clin Oral Investig. 2013;17:1773-83.

9. Xu L, Li J, Zhang M, Ai C, Wang L. Chinese authors do need CONSORT: reporting quality assessment for five leading Chinese medical journals. Contemp Clin Trials. 2008;29:727-31.

10. Hauptman JS, Chow DS, Martin NA, Itagaki MW. Research productivity in neurosurgery: trends in globalization, scientific focus, and funding. J Neurosurg. 2011;115:1262-72.

11. Grol R, Dalhuijsen J, Thomas S, Veld C, Rutten G, Mokkink H. Attributes of clinical guidelines that influence use of guidelines in general practice: observational study. BMJ. 1998;317:858-61.

12. Rehm J, Patra J. Different guidelines for different countries? On the scientific basis of low-risk drinking guidelines and their implications. Drug Alcohol Rev. 2012;31:156-61.

13. Liu W, Zhang Q, Ni M, Jia W, Jia G. Quality evaluation on china neurosurgical clinical practice guidelines. Nat Med J China. 2015;95:1122-6. article in Chinese.

14. Tang J, Chen C, Zhao Y. Neurosurgery center of beijing tiantan hospital, flagship of neurosurgery in china. World Neurosurgery. 2011;75:377-82.

15. Mao Y, Shi ZF, Zhou LF, Zhao Y. Huashan Hospital affiliated to Fudan University: spanning a century of history. World Neurosurgery. 2011;75:369-76.

16. Thomson Reuters Statement Regarding the San Francisco Declaration on Research Assessment [http://researchanalytics.thomsonreuters.com/ statement_re_sfdra/]

17. Seglen PO. Why the impact factor of journals should not be used for evaluating research. BMJ. 1997;314:498-502.

18. San Francisco Declaration on Research Assessment: Putting science into the assessment of research [http://www.ascb.org/dora/]

19. Zhao S, Lin $Y, X u$ W, Jiang W, Zha Z, Wang $P$, et al. Glioma-derived mutations in IDH1 dominantly inhibit IDH1 catalytic activity and induce HIF1alpha. Science. 2009;324:261-5.

20. Liu M, Wu B, Wang W-Z, Lee L-M, Zhang S-H, Kong L-Z. Stroke in China: epidemiology, prevention, and management strategies. Lancet Neurol. 2007;6:456-64.

21. Shi L, Cheng Z, Zhang J, Li R, Zhao P, Fu Z, et al. hsa-mir-181a and hsa-mir-181b function as tumor suppressors in human glioma cells. Brain Res. 2008;1236:185-93.

22. Fu Y-S, Cheng Y-C, Lin M-YA, Cheng H, Chu P-M, Chou S-C, et al. Conversion of human umbilical cord mesenchymal stem cells in Wharton's jelly to dopaminergic neurons in vitro: potential therapeutic application for Parkinsonism. Stem Cells. 2006;24:115-24.
23. Xiao G, Wei J, Yan W, Wang W, Lu Z. Improved outcomes from the administration of progesterone for patients with acute severe traumatic brain injury: a randomized controlled trial. Crit Care. 2008;12:R61.

24. Li J, Zhang N, Song L-B, Liao W-T, Jiang L-L, Gong L-Y, et al. Astrocyte elevated gene-1 is a novel prognostic marker for breast cancer progression and overall patient survival. Clin Cancer Res: Off J Am Assoc Cancer Res. 2008;14:3319-26.

25. Wan J, Cai W, Meng X, Liu E. Monodisperse water-soluble magnetite nanoparticles prepared by polyol process for high-performance magnetic resonance imaging. Chem Commun. 2007;21(47):5004-6.

26. Jiang J-Y, Gao G-Y, Li W-P, Yu M-K, Zhu C. Early indicators of prognosis in 846 cases of severe traumatic brain injury. J Neurotrauma. 2002;19:869-74.

27. Zhou X, Ren Y, Moore L, Mei M, You Y, Xu P, et al. Downregulation of miR-21 inhibits EGFR pathway and suppresses the growth of human glioblastoma cells independent of PTEN status. Lab Investig; J Technic Methods Pathol. 2010;90:144-55.

28. Zhang Y, Chao T, Li R, Liu W, Chen Y, Yan X, et al. MicroRNA-128 inhibits glioma cells proliferation by targeting transcription factor E2F3a. J Mol Med. 2009;87:43-51.

29. Hui AB, Lo KW, Yin XL, Poon WS, Ng HK. Detection of multiple gene amplifications in glioblastoma multiforme using array-based comparative genomic hybridization. Lab Investig; J Technic Methods Pathol. 2001;81:717-23.

30. Jiang J, Yu M, Zhu C. Effect of long-term mild hypothermia therapy in patients with severe traumatic brain injury: 1-year follow-up review of 87 cases. J Neurosurg. 2000;93:546-9.

31. Jiang B, Wang W, Chen H, Hong Z, Yang Q, Wu S, et al. Incidence and trends of stroke and its subtypes in China: results from three large cities. Stroke; J Cerebral Circ. 2006;37:63-8.

32. Huang $H$, Chen L, Wang H, Xiu B, Li B, Wang R, et al. Influence of patients' age on functional recovery after transplantation of olfactory ensheathing cells into injured spinal cord injury. Chin Med J. 2003;116:1488-91.

33. Wang C, Liu A, Zhang J, Sun B, Zhao Y. Surgical management of brain-stem cavernous malformations: report of 137 cases. Surg Neurol. 2003;59:444-54. discussion 454.

34. Wu J-S, Zhou L-F, Tang W-J, Mao Y, Hu J, Song Y-Y, et al. Clinical evaluation and follow-up outcome of diffusion tensor imaging-based functional neuronavigation: a prospective, controlled study in patients with gliomas involving pyramidal tracts. Neurosurgery. 2007;61:935-48. discussion 948-9.

35. Sui G, Yang X, Mei F, Hu X, Chen G, Deng X, et al. Poly-L-lactic acid/ hydroxyapatite hybrid membrane for bone tissue regeneration. J Biomed Mater Res A. 2007:82:445-54.

36. Yu C, Chen K, Zheng H, Guo X, Jia W, Li M, et al. Overexpression of astrocyte elevated gene-1 (AEG-1) is associated with esophageal squamous cell carcinoma (ESCC) progression and pathogenesis. Carcinogenesis. 2009;30:894-901.

37. Chen Y-S, Chang J-Y, Cheng C-Y, Tsai F-J, Yao C-H, Liu B-S. An in vivo evaluation of a biodegradable genipin-cross-linked gelatin peripheral nerve guide conduit material. Biomaterials. 2005;26:3911-8.

\section{Submit your next manuscript to BioMed Central and take full advantage of:}

- Convenient online submission

- Thorough peer review

- No space constraints or color figure charges

- Immediate publication on acceptance

- Inclusion in PubMed, CAS, Scopus and Google Scholar

- Research which is freely available for redistribution 